\title{
Using Benford's Law to Detect Anomalies in Electroencephalogram: An Application to Detecting Alzheimer's Disease
}

\author{
Santosh Tirunagari*†, Daniel Abasolo ${ }^{\ddagger}$, Aamo Iorliam ${ }^{\S}$, Anthony TS Ho*杖, and Norman Poh* \\ * Department of Computer Science. \\ $\ddagger$ Department of Mechanical Engineering Sciences. \\ $* \ddagger$ Faculty of Engineering and Physical Sciences, University of Surrey, Guildford, Surrey, United Kingdom GU2 7XH. \\ ${ }^{\dagger}$ Synoptic Technologies, Surrey Research Park, Guildford, Surrey, United Kingdom. \\ $\S$ Benue State University and Federal University of Agriculture, Makurdi, Nigeria. \\ I Wuhan University of Technology, China. \\ \| Tianjin University of Science and Technology, China.
}

\{santosh.tirunagari, d.abasolo, n.poh\}@ surrey.ac.uk.

\begin{abstract}
Alzheimer's disease (AD) is a neurodegenerative disease caused by the progressive death of brain cells over time. It represents the most frequent cause of dementia in the western world, and affects an individual's cognitive ability and psychological capacity. While clinical diagnoses of AD are made primarily on the basis of clinical evaluation and mental health tests, diagnostic certainty is only possible through necropsy. One non-invasive approach to investigating $\mathrm{AD}$ is to use electroencephalograms (EEGs), which reflect brain electrical activity and so can be used to detect electrical abnormalities in brain signals with non-invasive cranial surface electrodes. Generally EEGs in AD patients show a shift to lower frequencies in spectral analysis and display less complexity and contain more regular patterns compared to those of control subjects. Here we present a method for differentiating AD patients from healthy ones based on their EEG signals using Benford's law and support vector machines (SVMs) with a radial basis function (RBF) kernel. EEG signals from eleven AD and eleven age-matched controls were divided into artefact-free 5-sec epochs and used to train an SVM. 10 fold cross validation was performed at both the epochand subject-level to evaluate the importance of each electrode in discriminating between AD and healthy subjects. Substantive variability was seen across the different electrodes, with electrodes O1, $\mathrm{O} 2$ and $\mathrm{C} 4$ particularly being important. Performance across the electrodes was reduced when subject-level cross validation was performed, but relative performance across the electrodes was consistent with that found using epoch-level cross validation.
\end{abstract}

\section{INTRODUCTION}

Alzheimer's disease (AD) is the most frequent cause of dementia in the western world, and is caused by the progressive death of brain cells over time (neurodegeneration) affecting an individual's cognitive ability as well as influencing their psychological capacity [1]. Clinical diagnoses of AD are made primarily on the basis of medical history, psychiatric evaluation and memory and mental health tests [2], although an indisputable diagnosis is only possible through necropsy [3]. While symptoms and their severity vary from patient to patient, early diagnosis is vital for reducing their effects with available pharmacological treatments and through lifestyle adjustments [4].
Electroencephalograms (EEGs) have been used in the investigation of dementia for several decades [5]-[13]. Given that EEG signals reflect brain electrical activity from the cortex, they can be used to detect electrical abnormalities in brain signals with non-invasive cranial surface electrodes [14]. Generally EEGs in $\mathrm{AD}$ patients show a shift to lower frequencies in spectral analysis, which suggests a decreased cohesion of cognitive networks [14]. Moreover, AD patients' EEGs display less complexity and contain more regular patterns compared to those of control subjects [9], [14]-[16]. However, due to the intrinsic irregular and aperiodic nature of EEG signals, spectral analysis techniques may be insufficient for characterising the dynamics of the events underlying the EEG signals. Thus, additional techniques, have been developed, including nonlinear time series analysis [17] (particularly utilising entropy based methods [9], [15], [16]), for automatically distinguishing AD patients from control ones. Recently in [18] such methods were utilised for extracting features to a machine learning classifier. An in-depth review of findings distinguishing AD patients from control ones can be found in [10].

In 1881 Newcomb [19] observed that the first pages of the decadic logarithmic tables were more heavily worn than the last ones, indicating that the frequency of the first (most significant) digits decreases from 1 to 9. In 1938 Benford [20] analysed the distributions of the first digit from 20,000 different datasets and experimentally rediscovered and proved the behaviour observed by Newcomb. Benford's law has subsequently been shown to be both scale [21] and base invariant [22]. In 1978 Logan and Goudsmit [23] showed that tables containing random numbers follow Benford's law, while in 1996 Hill [24] showed if distributions are selected in a random (unbiased) manner and random samples taken from each of the distributions, then the significant digits of the combined sample will follow Benford's law. In 2002 Wallace [25] showed that when the mean of the data are greater than the median and the skewness is positive, the data are also likely to follow Benford's law. Benford's law has also been applied to problems in fields as diverse as 
accounting and neuroscience. In 1996 Nigrini [26] showed that manipulated tax data deviate from Benford's law, while Durtschi et al.'s [27] work demonstrated the effectiveness of Benford's law by using it to detect fraud in accounting data due to the data's deviation from Benford's law. Recently in 2014, Kreuzer et al. [28] for the first time showed that normal brain electrical activities analysed in form of EEG signals also follow Benford's law, and could be used to detect sevoflurane-induced signal modulations. Our study also identifies a Benford's law pattern in EEG data and could prove useful in developing novel automated methods for diagnosing disorders of the brain.

Our contributions can thus be summarised as follows:

- $\quad$ Novel use of first digit features (FDFs). Although FDFs from Benford's law have been used extensively as a feature descriptor in the fields of image and signal processing, their application to analysing AD patients' data are novel.

- Use of time-derivatives in EEG signals. We show that time-derivatives of EEG signals follow Benford's law, and that these time-derivatives can be used to discriminate between $\mathrm{AD}$ patients and control subjects.

- Proposal of FDF+SVM pipeline. FDFs can capture the descriptive probabilities of first digits from the EEG amplitudes. These features can be used as input to an SVM for classification.

- Rigorous performance evaluation for EEG data classification using half total error rates (HTER). Although HTER has been used extensively in the field of biometrics, its usage in biomedical applications is not very well known. HTER has the advantage of not being affected by the larger sample size of one class versus another because both types of errors are weighted equally, thus coercing equal contributions from both errors. Therefore, it has an advantage over traditional performance evaluation techniques such as the F-score or accuracy.

The organisation of the paper is as follows: In section II we present the Benford's law methodology as well as the evaluation criteria. In Section III we describe the dataset. Experiments and results are discussed in section IV, including data preprocessing. Finally, in section $\mathrm{V}$ we discuss the conclusions that can be drawn from the results.

\section{Methodology}

In this section we present Benford's law as applied to EEGs, and use SVMs with a radial basis function (RBF) kernel to distinguish between healthy and AD patients. The classification performance is evaluated by the half total error rate (HTER).

\section{A. Benford's Law}

Benford's law can be expressed mathematically as:

$$
\begin{aligned}
p(x) & =\log _{10}(x+1)-\log _{10}(x) \\
& =\log _{10}\left(\frac{x+1}{x}\right) \\
& =\log _{10}\left(1+\frac{1}{x}\right)
\end{aligned}
$$

where the leading digit $x \in\{1, \ldots, 9\}$ is the first digit of the number and $p(x)$ refers to the probability distribution of $x$.

Benford's law [29] follows the observation that the first significant digits are not uniformly distributed, but rather are heavily skewed towards the smaller digits for any real world distribution that spans across several orders of magnitude. There is neither an accepted proof [29], [30] nor a unified approach that explains the appearance of Benford's law in dynamical systems, number theory, statistics or real-world data [30]-[32].

When the most significant digit (MSD) of logarithms of the numbers are uniformly or randomly distributed, Benford's law can be expressed using the logarithmic scale such that the space between $x$ and $x+1$ on the logarithmic scale tallies with the expected Benford's law probability values. This implies that when considering values from 1 to 10 for a number $x$, for a number to start with digit 1 means that $1 \leq x<2$. In the same way, when taking $x=9$ it means that $9 \leq x<10$. When considering the $\log$ scale, this implies that $\log _{10} 1 \leq$ $x<\log _{10} 2$ will obtain a digit that has a MSD of 1 and that $\log \log _{10} 9 \leq \log _{10} x<\log _{10} 10$ will obtain a digit that has a MSD of 9. When the arithmetic scale is compared with the logarithmic scale, as shown in Figure 1, we can see that the blocks in the logarithmic scale are spaced differently when compared to the equally spaced blocks of the arithmetic scale.

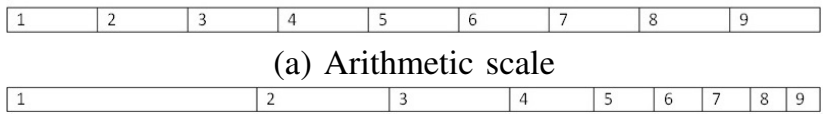

(b) logarithmic scale.

Fig. 1. Comparison between arithmetic and log scales.

One of the reasons for this can be explained by the intervals between the digits not being the same in the logarithmic scale. Whereas when considering the arithmetic scale there are equal intervals between the digits. Thus, if we consider an interval $\left[\log _{10} 1, \log _{10} 2\right]$ it gives $[0,0.3010]$ as compared to an interval of $\left[\log _{10} 9, \log _{10} 10\right]$ which gives $[0.9542425,1]$. This gives values of 0.30 for digit 1 and 0.05 for digit 9 , which tallies with the expected Benford's law probabilities of digits 1 and 9.

A typical distribution of digits according to Benford's law can be seen in Figure 2. Therefore, any data that closely follow this pattern follows the standard Benford's law [32].

\section{B. Classification Details}

The FDFs are given as input to the SVMs, which are used as they are known for their high classification accuracy. For the SVMs, an RBF kernel was used with kernel scale $\sigma=1$ and box constraint value set to 1 . Performance was evaluated using 10-fold cross validation run for 20 iterations, with the performance for each fold evaluated using the WER(HTER).

\section{Performance and Threshold Criteria}

Although the output of an SVM can be used to make a hard decision, we use a soft output, defined as the distance of a test sample from the SVM decision hyperplane. For all experiments, we label samples derived from AD patients' EEG 


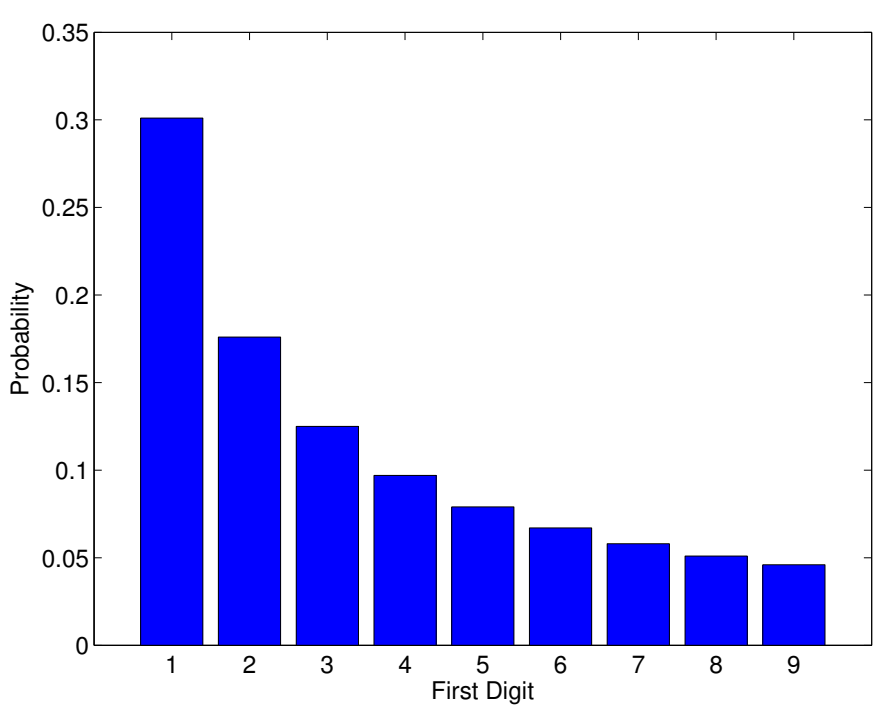

Fig. 2. Probability distribution of Benford's law

signals as positive, and those derived from the healthy subjects (i.e., the control) as negative.

Let $\mathcal{T}$ be the domain of the SVM output. A decision is made by comparing the SVM output $t \in \mathcal{T}$ with a decision threshold, $\Delta \in \mathcal{T}$, as follows:

$$
\operatorname{decision}(t)=\left\{\begin{array}{cl}
\text { positive } & \text { if } t>\Delta \\
\text { negative } & \text { otherwise, }
\end{array}\right.
$$

Let $\mathcal{T}_{+} \subset \mathcal{T}$ be the set of scores for the postive samples; and $\mathcal{T}_{-} \subset \mathcal{T}$, the negative samples. By applying the decision threshold $\Delta$, the sytem can commit two types of errors, namely, false rejection and false acceptance. Thier respective error rates are calculated as follow:

$$
\begin{aligned}
\operatorname{FRR}(\Delta) & \equiv P\left(t \leq \Delta \mid \mathcal{T}_{+}\right) \\
& \approx \frac{\left|\left\{t \in \mathcal{T}_{+}, t \leq \Delta\right\}\right|}{\left|\mathcal{T}_{+}\right|} \\
\operatorname{FAR}(\Delta) & \equiv 1-P\left(t \leq \Delta \mid \mathcal{T}_{-}\right), \\
& \approx \frac{\left|\left\{t \in \mathcal{T}_{-}, t>\Delta\right\}\right|}{\left|\mathcal{T}_{-}\right|}
\end{aligned}
$$

Note that the FRR is an increasing function of the decision threshold, whereas FAR is a decreasing function of it.

By plotting FRR versus FAR curve, one obtains a receiver's operating characteristic (ROC) curve. There are several useful point-estimate criteria that are useful to find the operational decision threshold, namely, equal error rate (EER), weighted error rate (WER) and its special case, half-total error rate (HTER).

The first one, EER, is the point where the two error rates cross each other:

$$
\operatorname{EER}\left(\Delta_{*}\right)=\operatorname{FAR}\left(\Delta_{*}\right)=\operatorname{FRR}\left(\Delta_{*}\right)
$$

where $\Delta_{*} \in \mathcal{T}$ is the unique decision threshold of the EER. In practice, the EER is found by searching for a threshold that minimises the absolute difference between the FAR and FRR.

$$
\Delta_{E E R}=\arg \min _{\Delta}|\operatorname{FAR}(\Delta)-\operatorname{FRR}(\Delta)|
$$

The second threshold-optimizing criterion is WER, and is defined as:

$$
W E R(\Delta)=\alpha \times \operatorname{FAR}(\Delta)+(1-\alpha) \times \operatorname{FRR}(\Delta)
$$

When $\alpha=\frac{1}{2}$, we have the HTER.

Throughout the experiments, we shall use EER to optimize the decision threshold and report performance in HTER.

The significant advantage of reporing the performance in HTER, is that it is not affected by the imbalance in the number of samples in each class as both types of errors are weighted equally, thus ensuring equal contribution from both errors (i.e. enforcing equal prior probability for both classes) [33], [34]. In our case, this is particularly desirable as the prior probability of a patient's EEG signal is difficult to estimate in practice. In the absence of any additional information, using equal prior probabilities is a reasonable option.

\section{DATASET}

The database used in this pilot study included 11 patients with a diagnosis of probable AD ( 5 men; 6 women; age: $72.5 \pm 8.3$ years, mean \pm standard deviation (SD)) and 11 agematched controls ( 7 men; 4 women; age: $72.8 \pm 6.1$ years, mean $\pm \mathrm{SD})$. The average Mini-Mental State Examination (MMSE) score for the AD patients was $13.1 \pm 5.9$ (mean $\pm \mathrm{SD}$ ). All control subjects had an MMSE score of 30 .

AD patients were recruited from the Alzheimer's Patients' Relatives Association of Valladolid (AFAVA), Spain. Informed consent was obtained for the recording of the EEG signals at the Hospital Clnico Universitario de Valladolid (Spain) using the international $10-20$ system and electrodes (Fp1, Fp2, F3, F4, C3, C4, P3, P4, O1, O2, F7, F8, T3, T4, T5, T6, Fz, Cz and $\mathrm{Pz}$ ) referenced to the linked ear lobes and with subjects sitting on a chair and with eyes closed. More than 5 minutes of EEG data were recorded for each subject using Oxford Instruments Profile Study Room 2.3.411 (Oxford, UK). A hardware lowpass filter with a cut-off frequency of $100 \mathrm{~Hz}$ was used before signals were sampled at $256 \mathrm{~Hz}$ and digitised with a 12-bit A-to-D converter. Five second epochs with little artefactual activity were selected for offline analysis as ASCII files by a specialist neurophysiologist, overseeing the recording of the EEGs. Artefacts included noise induced by eye movement and in no case electroencephalographic signs of sleep were observed. The total number of artefact-free epochs available for analysis was 9849 (5648 from AD patients and 4201 from control subjects). On average, $28.0 \pm 15.1$ epochs (meanSD) were available from each electrode and each subject.

\section{EXPERIMENTS AND RESULTS}

Our experiments in this study are enumerated below:

1) Do EEG signals follow Benford's law? As most natural data follows Benford's law, in this experiment we investigate whether EEG signals conform to this trend and follow Benford's law.

2) Effect of time-derivatives of EEG signals. In this experiment we investigate whether temporal derivative in EEG signals can improve the classification performance. 
3) Importance of electrode. In this experiment we investigate the performance obtained using each electrode, in terms of the WER(HTER).

4) Cross subject evaluation. Instead of performing cross validation at epoch level, where the training and test set may contain EEG signals from the same subjects, in this experiment we use subject-level cross validation. This ensures that each patient's data belongs to a single fold, thereby ensuring that we train on a set of subjects distinct from those used for testing.

For both experiments 3 and 4 above, the FDF features obtained from the time-derivatives in EEG signals identified in experiment 2 were used. We discuss the performance of our proposed pipeline and discuss the results in detail in the following subsections.

\section{A. Do EEG signals follow Benford's law?}

Although Kreuzer et al.'s [28] work showed that normal brain electrical activities analysed in the form of EEG signals follow Benford's law, our results from Fig. 3 show that most of the raw EEG signal did not follow Benford's law for both AD patients and the controls as their deviation from the Benford's law is greater.

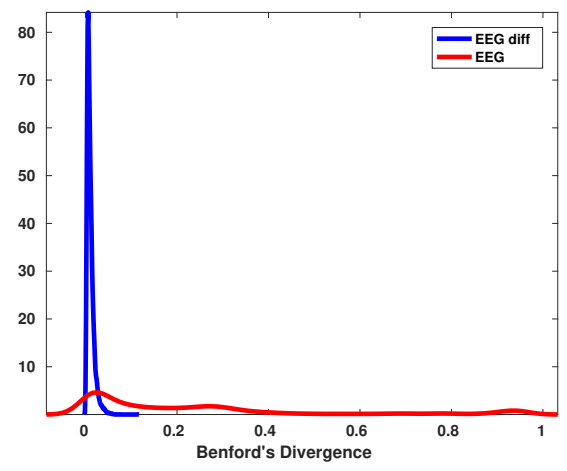

Fig. 3. $\chi^{2}$ divergence of first digit probabilities of all the electrodes from raw EEG signals as well as EEG with its time-derivatives. The kernel density values are calculated from 10332 epochs which includes AD patients as well as control ones. Greater divergence values indicate greater deviation from Benford's law. Hence lower the better.

A closer look into the first digit probabilities of electrode 01 on raw EEG signals reveal large amounts of outlier points for both $\mathrm{AD}$ patients as well as for control ones (Fig. 4).

The classification performance achieved was approximately 30 percent in WER(HTER) across all the electrodes, as shown in Table I.

Since most of the raw EEG signals did not follow Benford's law (Fig. 4) and the result yielded a high WER(HTER) (Table I), we wanted to see if time-derivatives in EEG signals follow Benford's law. The motivation for this comes from our recent work in detecting intrusion attacks from network traffic flows [31], where we discuss why time-derivatives in data may follow Benford's law. The rationale of this is that by taking the time-derivative, the magnitudes are cancelled out and so we look at the first derivatives of the EEG signal. The signals in raw EEG may be slower when compared to time-derivative in EEG signals.
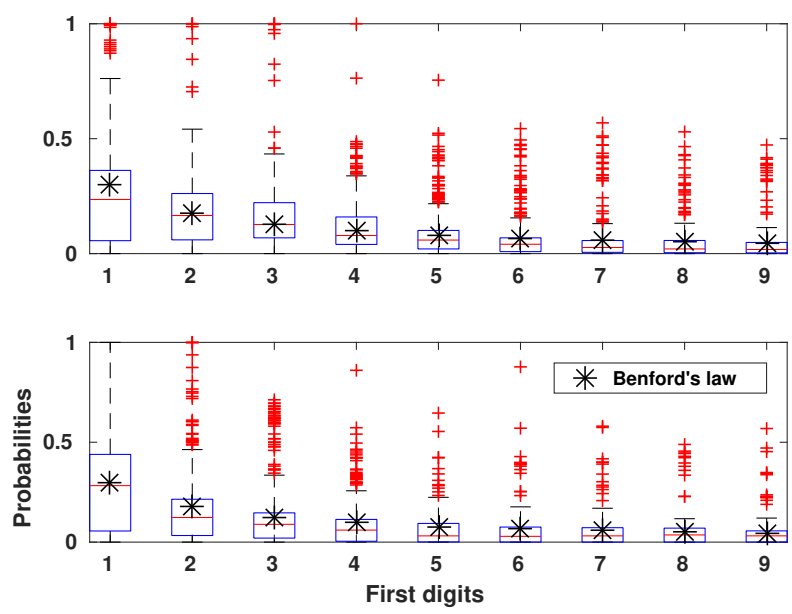

Fig. 4. First digit probabilities of electrode $\mathbf{O 1}$ on raw EEG signals. (Top) Alzheimer's patients and (bottom) control subjects. * in the Figure represents the true probabilities of first digits according to Benford's law. There are 364 and 263 measurements for each bar across all the electrodes for AD patients and control subjects respectively.

TABLE I. MEANS AND STANDARD DEVIATIONS OF THE HALF TOTAL ERROR RATE OVER 20 RUNS OF 10 FOLD CROSS VALIDATION.

\begin{tabular}{|c|c|c|}
\hline Electrode & \multicolumn{2}{|c|}{ WER(HTER) (mean \pm std) } \\
\hline & EEG signals & time-derivatives of EEG \\
\hline C3 & $0.3136 \pm 0.0407$ & $0.1925 \pm 0.0313$ \\
\hline C4 & $0.2592 \pm 0.0326$ & $\mathbf{0 . 1 4 3 2} \pm \mathbf{0 . 0 3 0 0}$ \\
\hline F3 & $0.2793 \pm 0.0375$ & $0.1549 \pm 0.0287$ \\
\hline F4 & $0.3080 \pm 0.0319$ & $0.2699 \pm 0.0417$ \\
\hline F7 & $0.3358 \pm 0.0391$ & $0.2535 \pm 0.0395$ \\
\hline F8 & $0.2676 \pm 0.0364$ & $0.1975 \pm 0.0305$ \\
\hline Fp1 & $0.3016 \pm 0.0393$ & $0.1956 \pm 0.0301$ \\
\hline Fp2 & $0.2807 \pm 0.0348$ & $0.1976 \pm 0.0341$ \\
\hline O2 & $0.3435 \pm 0.0395$ & $0.1854 \pm 0.0326$ \\
\hline O1 & $0.3219 \pm 0.0367$ & $\mathbf{0 . 1 4 4 1} \pm \mathbf{0 . 0 2 8 9}$ \\
\hline P3 & $0.3465 \pm 0.0370$ & $0.1693 \pm 0.0277$ \\
\hline P4 & $0.3296 \pm 0.0323$ & $0.2279 \pm 0.0327$ \\
\hline T3 & $0.2825 \pm 0.0389$ & $0.2973 \pm 0.0483$ \\
\hline T4 & $0.2775 \pm 0.0410$ & $0.1957 \pm 0.0299$ \\
\hline T5 & $0.2715 \pm 0.0380$ & $0.1803 \pm 0.0332$ \\
\hline T6 & $0.3372 \pm 0.0345$ & $0.2454 \pm 0.0316$ \\
\hline
\end{tabular}
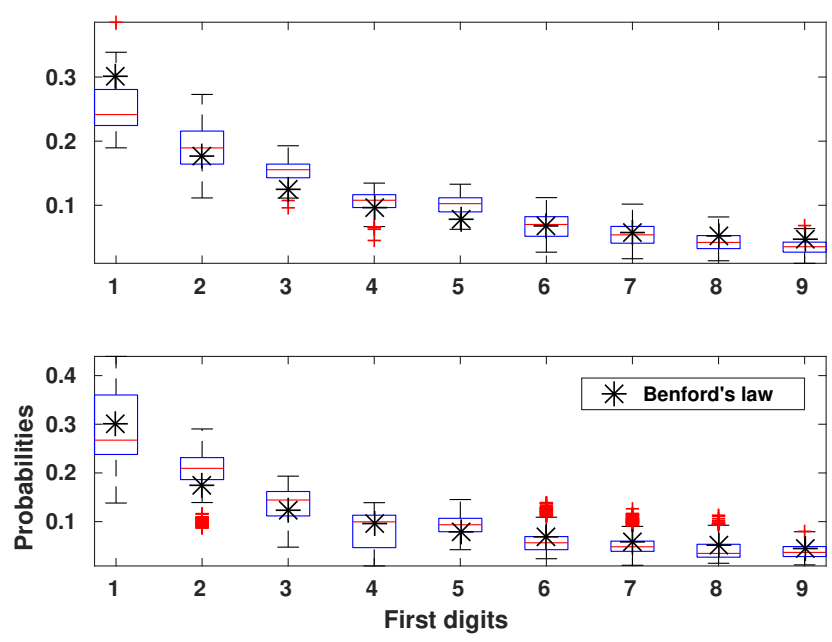

Fig. 5. First digit probabilities of time-derivatives in electrode 01 EEG signals. (Top) AD patients and (bottom) control subjects.

\section{B. Time-derivatives of EEG signals vs Benford's law}

Results from Fig. 3 show that most of the time-derivatives in EEG signals follow Benford's law for both AD patients and 
the controls as their deviation from the Benford's law is lower when compared to raw EEG signals. Indeed from Fig. 5 we can see that time-derivatives in EEG signal epochs contained fewer outlier points suggesting that they have followed Benford's law in both AD patients and controls. The results using the WER(HTER) criterion showed approximately a 10 percentage point improvement in performance over the results obtained using raw EEG signals (Table I).

\section{Importance of electrodes}

From Table I, we can see that electrode C4 gave a WER(HTER) of $0.1432 \pm 0.03$, which is the minimum when compared to other electrodes followed by 01 with a WER(HTER) of $0.1441 \pm 0.03$. Both electrodes showed consistently low WER(HTER) values, as can be observed from Fig. 6.

\section{Subject-level cross-validation}

In the previous experiments, we evaluated performance at the epoch level, where EEG signals from a single subject could be contained in both the training and test sets. We suspect that this will have positively biased the performance of our classification model. Therefore, we created 10 cross validation folds at the subject level, as shown in Table II, to enable training on one set of subjects and testing the performance on a separate set of subjects. At least one subject with $\mathrm{AD}$ is always present in the test set.

TABLE II. NUMBER OF SUBJECTS CONSIDERED IN THE TRAINING AND TEST SETS ACROSS 10 FOLDS.

\begin{tabular}{|l|l|l|l|l|l|l|l|l|l|l|}
\hline Train size & 20 & 19 & 19 & 20 & 20 & 20 & 20 & 20 & 20 & 20 \\
\hline Test size & 2 & 3 & 3 & 2 & 2 & 2 & 2 & 2 & 2 & 2 \\
\hline
\end{tabular}

The results from Table III show an increase in the WER(HTER) across all electrodes. This proves our hypothesis that the classification model was positively biased when cross validation was not performed at the subject level.

TABLE III. MEANS AND STANDARD DEVIATIONS OF WEIGHTED ERROR RATES OVER 20 RUNS OF 10 FOLD CROSS VALIDATION PERFORMED AT THE SUBJECT LEVEL.

\begin{tabular}{|c|c|}
\hline Electrode & WER(HTER) $($ mean \pm std) \\
\hline C3 & $0.3660 \pm 0.1431$ \\
\hline C4 & $\mathbf{0 . 2 5 3 0} \pm \mathbf{0 . 1 5 4 5}$ \\
\hline F3 & $0.2813 \pm 0.1422$ \\
\hline F4 & $0.3555 \pm 0.1336$ \\
\hline F7 & $0.3572 \pm 0.1344$ \\
\hline F8 & $0.3132 \pm 0.1600$ \\
\hline Fp1 & $0.2926 \pm 0.1683$ \\
\hline Fp2 & $0.3121 \pm 0.1600$ \\
\hline O2 & $0.2567 \pm 0.1561$ \\
\hline O1 & $\mathbf{0 . 2 2 2 2} \pm \mathbf{0 . 1 8 6 6}$ \\
\hline P3 & $0.3262 \pm 0.1479$ \\
\hline P4 & $0.3563 \pm 0.1493$ \\
\hline T3 & $0.4367 \pm 0.0842$ \\
\hline T4 & $0.2804 \pm 0.1449$ \\
\hline T5 & $0.2876 \pm 0.1392$ \\
\hline T6 & $0.3886 \pm 0.1454$ \\
\hline
\end{tabular}

Although we see an increase in the WER(HTER) across all the electrodes, the results in Fig. I are consistent with the results shown in Fig. III. Electrodes $\mathbf{O 1}$ and $\mathbf{C 4}$ still proved to be the best at discriminating between the AD patients and the controls. With the relevance of this to a neurophysiological point of view, electrode $\mathbf{O 2}$ is one of the electrodes we tend to see differences between in both groups, but $\mathbf{C 4}$ is not usually flagged up with the non-linear methods.

\section{Discussions AND CONCLUSIONS}

The quick and accurate detection of $\mathrm{AD}$ is vital for slowing the progression of the disease, mitigating its symptoms and extending the patients' quality of life years. However, the extensive medical evaluation and testing needed before a diagnosis can be made can leave long periods between the onset of symptoms and a final diagnosis, with diagnostic certainty still not being possible without post-mortem examination. A quick and accurate method for identifying patients with $\mathrm{AD}$ would therefore fill an unmet clinical need. To this end we present a method for discriminating between $\mathrm{AD}$ and healthy patients based on their EEG signals using first digit features from Benford's law and SVMs with an RBF kernel.

We found that mean HTER varies substantively between electrodes both in the epoch- and subject-level experiments. In the case of the epoch-level experiments, electrodes covering the frontal and temporal lobes were consistently less useful in discriminating between AD and healthy subjects. However, this pattern was not observed in the subject-level experiments, with no discernible trend in performance across lobes or hemispheres. Despite this, electrodes 01, C4 and 02 showed high discriminating power across both experiments. With the relevance of this from a neurophysiological point of view, electrode $\mathbf{O 2}$ is one of the electrodes we tend to see differences between both groups, but $\mathbf{C 4}$ is not usually flagged up with the non-linear methods [9], [15]-[17].

\section{ACKNOWLEDGEMENT}

The authors would like to thank Dr. Simon C Bull for his support and assistance with this study. 'ST' and 'NP' have benefited from the Medical Research Council (MRC)-funded project 'Modelling the Progression of Chronic Kidney Disease' $(\mathrm{R} / \mathrm{M} 023281 / 1)^{1}$.

\section{REFERENCES}

[1] M. Graeber, S. Kösel, R. Egensperger, R. Banati, U. Müller, K. Bise, P. Hoff, H. Möller, K. Fujisawa, and P. Mehraein, "Rediscovery of the case described by alois alzheimer in 1911: historical, histological and molecular genetic analysis," Neurogenetics, vol. 1, no. 1, pp. 73-80, 1997.

[2] G. M. McKhann, D. S. Knopman, H. Chertkow, B. T. Hyman, C. R. Jack, C. H. Kawas, W. E. Klunk, W. J. Koroshetz, J. J. Manly, R. Mayeux et al., "The diagnosis of dementia due to alzheimers disease: Recommendations from the national institute on aging-alzheimers association workgroups on diagnostic guidelines for alzheimer's disease," Alzheimer's \& dementia, vol. 7, no. 3, pp. 263-269, 2011.

[3] D. S. Knopman, S. T. DeKosky, J. Cummings, H. Chui, J. CoreyBloom, N. Relkin, G. Small, B. Miller, and J. Stevens, "Practice parameter: Diagnosis of dementia (an evidence-based review) report of the quality standards subcommittee of the american academy of neurology," Neurology, vol. 56, no. 9, pp. 1143-1153, 2001.

[4] B. Reisberg, R. Doody, A. Stöffler, F. Schmitt, S. Ferris, and H. J. Möbius, "Memantine in moderate-to-severe alzheimer's disease," New England Journal of Medicine, vol. 348, no. 14, pp. 1333-1341, 2003.

[5] L. A. Coben, W. L. Danziger, and L. Berg, "Frequency analysis of the resting awake eeg in mild senile dementia of alzheimer type," Electroencephalography and clinical neurophysiology, vol. 55, no. 4, pp. 372-380, 1983.

\footnotetext{
${ }^{1}$ www.modellingCKD.org
} 

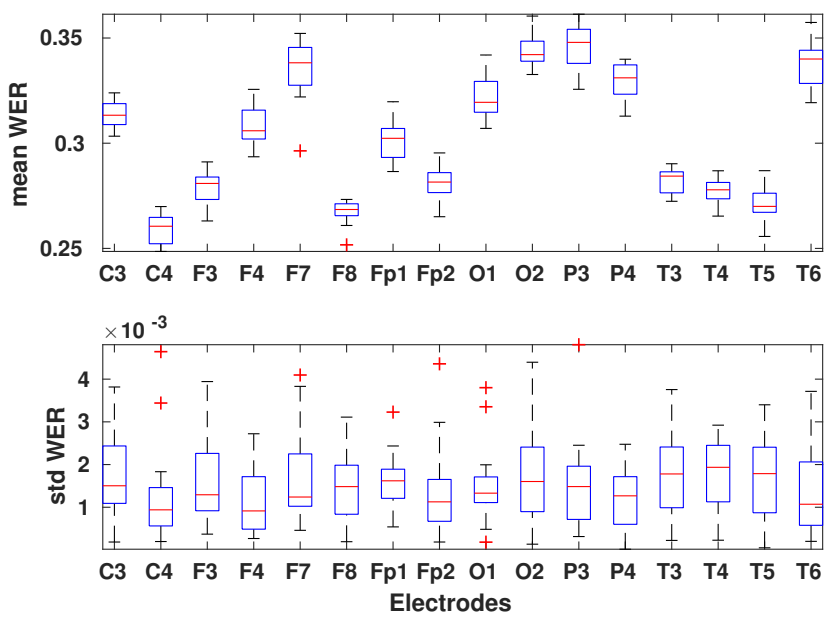

(a)
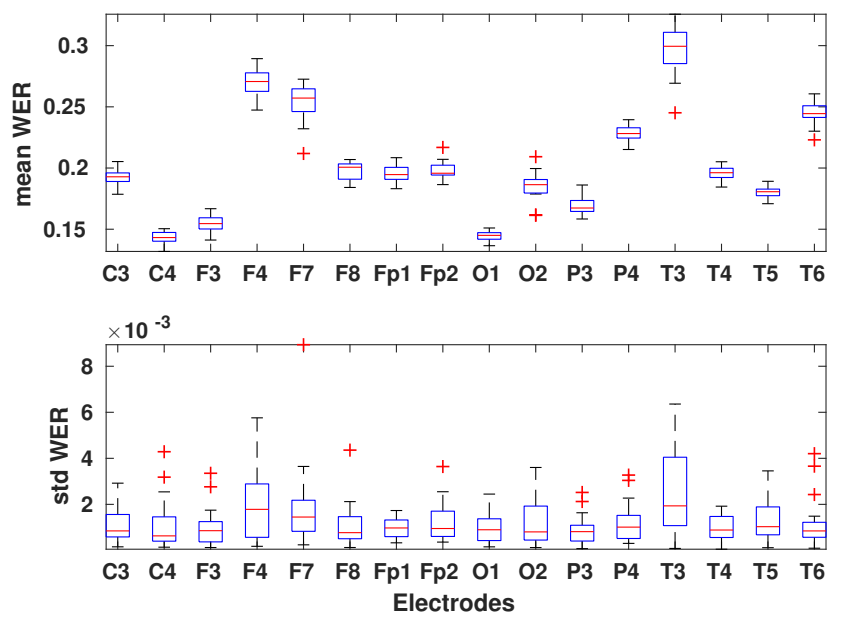

(b)

Fig. 6. Mean and standard deviations of the weighted error rate over 20 runs of 10 fold cross validation. (a) raw EEG signals (b) time-derivatives in EEG signals.

[6] F. Miraglia, F. Vecchio, and P. M. Rossini, "Searching for signs of aging and dementia in eeg through network analysis," Behavioural brain research, vol. 317, pp. 292-300, 2017.

[7] R. Gandelman-Marton, S. Aichenbaum, E. Dobronevsky, M. Khaigrekht, and J. M. Rabey, "Quantitative eeg after brain stimulation and cognitive training in alzheimer disease," Journal of Clinical Neurophysiology, vol. 34, no. 1, pp. 49-54, 2017.

[8] M. Chochoi, L. Tyvaert, P. Derambure, and W. Szurhaj, "Is long-term electroencephalogram more appropriate than standard electroencephalogram in the elderly?" Clinical Neurophysiology, vol. 128, no. 1, pp. 270-274, 2017.

[9] D. Abásolo, R. Hornero, C. Gómez, M. García, and M. López, “Analysis of eeg background activity in alzheimer's disease patients with lempelziv complexity and central tendency measure," Medical Engineering \& Physics, vol. 28, no. 4, pp. 315-322, 2006.

[10] J. Dauwels, F. Vialatte, and A. Cichocki, "Diagnosis of alzheimer's disease from eeg signals: where are we standing?" Current Alzheimer Research, vol. 7, no. 6, pp. 487-505, 2010.

[11] J. Dauwels, F. Vialatte, T. Musha, and A. Cichocki, "A comparative study of synchrony measures for the early diagnosis of alzheimer's disease based on eeg," NeuroImage, vol. 49, no. 1, pp. 668-693, 2010.

[12] J. Dauwels, K. Srinivasan, M. Ramasubba Reddy, T. Musha, F.-B. Vialatte, C. Latchoumane, J. Jeong, and A. Cichocki, "Slowing and loss of complexity in alzheimer's eeg: two sides of the same coin?" International journal of Alzheimers disease, vol. 2011, 2011.

[13] D. Labate, F. La Foresta, G. Morabito, I. Palamara, and F. C. Morabito, "Entropic measures of eeg complexity in alzheimer's disease through a multivariate multiscale approach," IEEE Sensors Journal, vol. 13, no. 9, pp. 3284-3292, 2013.

[14] J. Jeong, "Eeg dynamics in patients with alzheimer's disease," Clinical neurophysiology, vol. 115, no. 7, pp. 1490-1505, 2004.

[15] H. Azami, D. Abásolo, S. Simons, and J. Escudero, "Univariate and multivariate generalized multiscale entropy to characterise eeg signals in alzheimers disease," Entropy, vol. 19, no. 1, p. 31, 2017.

[16] D. Abásolo, R. Hornero, P. Espino, J. Poza, C. I. Sánchez, and R. de la Rosa, "Analysis of regularity in the eeg background activity of alzheimer's disease patients with approximate entropy," Clinical Neurophysiology, vol. 116, no. 8, pp. 1826-1834, 2005.

[17] C. J. Stam, "Nonlinear dynamical analysis of eeg and meg: review of an emerging field," Clinical neurophysiology, vol. 116, no. 10, pp. 22662301, 2005.

[18] S. Simons, D. Abasolo, and J. Escudero, "Classification of alzheimer's disease from quadratic sample entropy of electroencephalogram," Healthcare technology letters, vol. 2, no. 3, pp. 70-73, 2015.

[19] S. Newcomb, "Note on the frequency of use of the different digits in natural numbers," American Journal of Mathematics, vol. 4, no. 1, pp. 39-40, 1881.

[20] F. Benford, "The law of anomalous numbers," Proceedings of the American Philosophical Society, vol. 78, pp. 551-572, 1938.

[21] R. S. Pinkham, "On the distribution of first significant digits," The Annals of Mathematical Statistics, vol. 32, no. 4, pp. 1223-1230, 1961.

[22] T. P. Hill, "Base-invariance implies benfords law," Proceedings of the American Mathematical Society, vol. 123, no. 3, pp. 887-895, 1995.

[23] J. L. Logan and S. A. Goudsmit, "The first digit phenomenon," Proceedings of the American Philosophical Society, vol. 122, no. 4, pp. 193-197, 1978.

[24] T. P. Hill, "A statistical derivation of the significant-digit law," Statistical Science, vol. 10, pp. 354-363, 1996.

[25] W. A. Wallace, "Assessing the quality of data used for benchmarking and decision-making," The Journal of Government Financial Management, vol. 51, no. 3, p. 16, 2002.

[26] M. J. Nigrini, "A taxpayer compliance application of benford's law," The Journal of the American Taxation Association, vol. 18, no. 1, p. 72, 1996.

[27] C. Durtschi, W. Hillison, and C. Pacini, "The effective use of benford's law to assist in detecting fraud in accounting data," Journal of forensic accounting, vol. 5, no. 1, pp. 17-34, 2004.

[28] M. Kreuzer, D. Jordan, B. Antkowiak, B. Drexler, E. F. Kochs, and G. Schneider, "Brain electrical activity obeys benford's law," Anesthesia \& Analgesia, vol. 118, no. 1, pp. 183-191, 2014.

[29] A. Berger and T. P. Hill, "Benfords law strikes back: No simple explanation in sight for mathematical gem," The Mathematical Intelligencer, vol. 33, no. 1, pp. 85-91, 2011.

[30] R. M. Fewster, "A simple explanation of benford's law," The American Statistician, 2012.

[31] A. Iorliam, S. Tirunagari, A. T. Ho, S. Li, A. Waller, and N. Poh, "'flow size difference" can make a difference: Detecting malicious tcp network flows based on benford's law," arXiv preprint arXiv:1609.04214, 2016.

[32] A. Iorliam, A. T. Ho, N. Poh, S. Tirunagari, and P. Bours, "Data forensic techniques using benford's law and zipf's law for keystroke dynamics," in Biometrics and Forensics (IWBF), 2015 International Workshop on. IEEE, 2015, pp. 1-6.

[33] S. Tirunagari, N. Poh, D. Windridge, A. Iorliam, N. Suki, and A. T. Ho, "Detection of face spoofing using visual dynamics," IEEE transactions on information forensics and security, vol. 10, no. 4, pp. 762-777, 2015.

[34] S. Tirunagari, N. Poh, M. Bober, and D. Windridge, "Windowed dmd as a microtexture descriptor for finger vein counter-spoofing in biometrics," in Information Forensics and Security (WIFS), 2015 IEEE International Workshop on. IEEE, 2015, pp. 1-6. 